\title{
Acceso a servicios de salud en mujeres transgénero de la ciudad de Cali, Colombia
}

\author{
Access to Health Services for Transgender Women in the City of Cali, \\ Colombia
}

\section{Acesso a serviços de saúde para mulheres transgêneros na cidade de Cali, Colômbia}

Claudia Marcela Domínguez, Psi., Esp., MSc. *

Sandra Viviana Ramírez, Psi., Esp., MSc. **

Marcela Arrivillaga-Quintero, Psi., Esp., MSc., PhD. ***

\section{Resumen}

Introducción: La población transgénero enfrenta retos en cuanto a la garantía del derecho a la salud y el acceso efectivo a los servicios de salud en todos los países. Objetivo: Describir y analizar el acceso a los servicios de salud preventivos, curativos, de atención específica, y asociados al gasto de bolsillo, en mujeres transgénero de Cali, Colombia. Metodología: Estudio observacional de tipo transversal con muestreo probabilístico. Se aplicó una adaptación de la Encuesta de Acceso a Servicios de Salud para Hogares Colombianos a un total de 109 personas que se auto identificaron como transgénero, mayores de 18 años. Se efectuó un análisis univariado de la totalidad de las variables evaluadas. Resultados: Si bien el $71.6 \%$ de las mujeres transgénero encuestadas se encontraban cubiertas por el sistema de salud, esto no garantiza el acceso oportuno a los servicios. Existe debilidad institucional por parte de las Entidades Promotoras de Salud (EPS) para promover servicios preventivos; el acceso a consulta con especialista y a medicamentos tuvo resultados desfavorables en

\begin{abstract}
oportunidad y calidad; no se atienden necesidades relacionadas con su identidad de género ni procesos de transformación corporal. Discusión: Tal como se ha reportado en otros estudios para población general, los hallazgos son coherentes con la estructura del sistema de salud colombiano que está diseñado para invertir los recursos en la atención de la enfermedad y no en la prevención. Conclusiones: Se requieren protocolos de atención diferencial dados los riesgos para personas transgénero por falta de atención médica y psicosocial. De esta manera, se garantiza el pleno derecho a la salud y el respeto por el libre desarrollo de la personalidad, establecido constitucionalmente en el país. [Domínguez CM, Ramírez SV, Arrivillaga-Quintero M. Acceso a servicios de salud en mujeres transgénero de la ciudad de Cali, Colombia. MedUNAB 2017-2018; 20(3): 296-309].
\end{abstract}

Palabras clave: Personas Transgénero; Accesibilidad a los Servicios de Salud; Identidad de Género; Acceso Universal a Servicios de Salud; Colombia.

\footnotetext{
* Psicóloga, especialista en Gerencia Social, magíster en Salud Pública, Pontificia Universidad Javeriana, Cali, Valle del Cauca, Colombia

** Psicóloga, especialista en Desarrollo Humano y Organizacional, magíster en Salud Pública, Pontificia Universidad Javeriana, Valle del Cauca, Cali, Colombia ***Psicóloga,especialización WHO/PAHO, magíster en Educación, doctorado en Salud Pública. Profesora titular, Departamento de Salud Pública y
} Epidemiología, Facultad de Ciencias de la Salud, Pontificia Universidad Javeriana, Cali, Valle del Cauca, Colombia.

Correspondencia: Marcela Arrivillaga-Quintero. Calle 18 No. 118-250, Cali, Colombia. Teléfono: +57 (2) 3218200 ; extensión: 8681. E-mail: marceq@javerianacali.edu.co 


\section{Abstract}

Introduction: Transgender population faces challenges in terms of guaranteeing the rights to health and an effective access to health services in every country. Objective: To describe and analyze the access to preventive, healing, specific care, and associated out-of-pocket health services for transgender women in Cali, Colombia. Methodology: An observational cross-sectional study with probability sampling. An adaptation of the Survey on Access to Health Services for Colombian Homes was applied to a total of 109 people who identified themselves as transgender, and all of them were over 18. A univariate analysis of all variables evaluated was carried out. Results: Although $71.6 \%$ of transgender women surveyed were covered by the health system, this percentage does not guarantee timely access to services. There is an institutional weakness regarding to the Health Promoting Entities (EPS) to promote preventive services; the access to consultation with a specialist and medicines had unfavorable results in opportunity and quality; also, the needs related to their gender identity or processes of corporal transformation are not treated. Discussion: As reported in other studies, for the population in general, the findings are coherent with the Colombian health system structure which is designed to invest resources in treating diseases but not preventing them. Conclusions: Differential care protocols are required given the risks for transgender people due to the lack of medical and psychosocial care. In this way, the full right to health, and the respect for the free development of personality are guaranteed, which were constitutionally established in the country. [Domínguez CM, Ramírez SV, Arrivillaga-Quintero $M$. Access to Health Services for Transgender Women in the City of Cali, Colombia. MedUNAB 2017-2018; 20(3): 296-309].

Keywords: Transgender Persons; Health Services Accessibility; Gender Identity; Universal Access to Health Care Services; Colombia.

\section{Introduction}

La garantía del derecho fundamental a la salud está directamente relacionada con el acceso efectivo a los servicios de salud, con la forma en que están organizados y con la oportunidad e integralidad de la atención, tal como se ha declarado en la Ley 1751 de 2015 o Ley Estatutaria en salud. A pesar de esto, 22 años después de la entrada de la reforma a la salud en Colombia y dos años después de la entrada de la Ley Estatutaria, el sistema sigue teniendo retos para garantizar este derecho, y el acceso real y efectivo a los servicios.

La mayoría de los estudios sobre equidad en el acceso a los servicios de salud en Colombia defienden la tesis de que como resultado de la Ley 100 de 1993 (1) puede considerarse que hay mayor equidad debido a la ampliación de la cobertura, en especial de la población más pobre. Sin embargo, datos regionales o por ciudades específicas como

\section{Resumo}

Introdução: a população transgênera enfrenta desafios em relação à garantia do direito à saúde e ao acesso efetivo aos serviços de saúde, em todos os países. Objetivo: Descrever e analisar o acesso aos cuidados preventivos, curativos e específicos, como também, aos relacionados com os serviços de saúde proprios para as mulheres transexuais em Cali, Colômbia. Metodologia: estudo transversal de observação com amostragem probabilística. Uma adaptação da Pesquisa de Acesso aos Serviços de Saúde para Casas Colombianas, foi aplicada a um total de 109 pessoas que se identificaram como transexuais, com mais de 18 anos de idade. Uma análise univariada, de todas as variáveis avaliadas, foi realizada. Resultados: Apesar de $71,6 \%$ das mulheres transexuais entrevistadas, serem cobertas pelo sistema de saúde, isso não garante o acesso oportuno aos serviços. Existe uma fraqueza institucional por parte das Entidades Promotoras da Saúde (EPS), para promover serviços preventivos; o acesso à consulta com especialistas e medicamentos teve resultados desfavoráveis em oportunidade e qualidade; não são atendidas às necessidades relacionadas à sua identidade de gênero ou processos de transformação corporal. Discussão: conforme relatado em outros estudos para a população em geral, os achados são coerentes com a estrutura do sistema de saúde colombiano, projetado para investir recursos no cuidado da doença e não na prevenção. Conclusões: os protocolos de cuidados diferenciais são necessários, dado os riscos para pessoas transexuais devido à falta de cuidados médicos e psicossociais. Desta forma, é garantido o pleno direito à saúde e o respeito pelo livre desenvolvimento da personalidade, constitucionalmente estabelecido no país. [Domínguez CM, Ramírez SV, Arrivillaga-Quintero M. Acesso a serviços de saúde para mulheres transgêneros na cidade de Cali, Colômbia. MedUNAB 2017-2018; 20(3): 296-309].

Palavras-Chave: Pessoas Transgênero; Acesso aos Serviços de Saúde; Identidade de Gênero; Acesso Universal a Serviços de Saúde; Colômbia.

Bogotá, señalan la persistencia de inequidades en el acceso tanto en población cubierta como la no cubierta por el sistema (2). Estas inequidades se incrementan en sectores de la población que enfrentan barreras de tipo geográfico, normativo, administrativo, cultural y de oferta (3), y pueden ser más visibles en los grupos sociales diversos por sus características étnicas, sociales o de orientación sexual.

Entre las situaciones que tienen que enfrentar las diversas minorías sexuales en cuanto al acceso a servicios de salud se encuentran la discriminación, estigma y menosprecio a que son sometidos; estos grupos llegan a categorizarse como "pintorescos", atípicos y extraños (4). A su vez, enfrentan mayor vulnerabilidad social, económica y de salud, pues históricamente han experimentado desventajas e inequidades en el acceso a bienes y servicios que puedan favorecer su bienestar integral y su calidad de vida.

Uno de los grupos diversos que ha venido reconfigurando su lucha por la garantía de derechos son los movimientos de 
Lesbianas, Gay, Transgénero, Bisexuales e Intersexuales (LGTBI). En particular, las personas transgénero son aquellas cuya identidad y expresión de género no corresponde con las normas y expectativas sociales tradicionalmente asociadas con su sexo asignado al nacer (5). Muchos de los conceptos relativos al género que se utilizan en las culturas occidentales se basan en una concepción binaria del sexo, que considera que existen dos polos opuestos: hombre-mujer, masculino-femenino y hembra-macho. No obstante, las personas transgénero son una población de individuos que cruzan o trascienden categorías de género culturalmente definidas $(5,6)$. Las identidades transgénero incluyen el tránsito de hombre a mujer y de mujer a hombre (mujeres y hombres, que feminizan o masculinizan sus cuerpos a través de la terapia hormonal o cirugía), travestis (que utilizan vestimenta típicamente usada por otro género como medio de expresión erótica), Drag Queens/Kings (imitadores femeninos o masculinos), y otras personas que se consideran Queer (7). En Colombia y en el mundo las personas transgénero representan una de las poblaciones menos favorecidas, por la carga de enfermedades que experimentan, el estigma y la discriminación $(8,9)$. Esto agudiza los riesgos y obstaculiza el disfrute pleno de derechos en general, y por supuesto, del derecho a la salud.

En cuanto a estadísticas, algunas cifras señalan que a nivel global existen alrededor de 1,100.000 hombres que se sienten mujeres y 1,200.000 mujeres que se consideran hombres (10). En 2013 se realizó la primera revisión de estudios publicados en mujeres transgénero, mostrando afectación consistente en 15 países del mundo, entre ellos cuatro de América Latina: Argentina, Brasil, El Salvador y Perú (4). En Colombia, en el año 2012 se reportó una prevalencia de VIH para este grupo de 17\% para Bogotá, 10\% para Medellín, 13\% Barranquilla y 19\% Cali (10).

Si bien es cierto que la tendencia en la investigación ha estado concentrada en población transgénero y Virus de Inmunodeficiencia humana/Síndrome de la Inmunodeficiencia Adquirida (VIH/SIDA) (10), se conoce que existen otra serie de riesgos que afectan su salud. La automedicación de hormonas y los procedimientos estéticos no realizados por personal médico (8), el consumo de sustancias psicoactivas, la vinculación laboral al trabajo informal, que en la mayoría de los casos es trabajo sexual con sus consecuentes implicaciones, y la violencia a la que se exponen a causa del estigma y la discriminación (11-13), son otras condiciones de vida que ameritan un abordaje diferencial en cuanto al acceso a los servicios de salud. No obstante, este fenómeno, aún en contextos donde las prevalencias de VIH/SIDA son elevadas, no ha sido analizado en Colombia. Esto impide avanzar en la comprensión de las necesidades de esta población y por ende proponer estrategias de intervención efectivas en promoción, prevención y atención.

Considerando lo anterior, este estudio tuvo como objetivo describir y analizar el acceso a los servicios de salud preventivos, curativos, de atención específica y gasto de bolsillo, en mujeres transgénero en la ciudad de Cali, Colombia, e identificar prioridades específicas en salud para formular recomendaciones que promuevan la atención integral de esta población.

\section{Metodología}

Se realizó un estudio de tipo cuantitativo, con diseño observacional, descriptivo-transversal. Previamente aprobado por el Comité de Ética de la Investigación de la Facultad de Ciencias de la Salud de la Pontificia Universidad Javeriana, Cali, Colombia. Se utilizó un muestreo probabilístico, por conglomerados y polietapico. De acuerdo con el número de la población estimada de 1,267 mujeres transgénero para la ciudad de Cali (14). Se calculó una muestra de 109 mujeres transgénero, que fueron ubicadas en cinco zonas de convergencia, que para el caso del estudio fueron definidas como paradas o sitios de encuentro y tránsito. Estas fueron: 5 clubes y reservados, 5 peluquerías, 12 zonas de tolerancia para el ejercicio del trabajo sexual y convergencia de socialización, 2 fundaciones, y 5 cyber.

La selección de las participantes se desarrolló por etapas sucesivas a través de un muestreo aleatorio. En cada una de las 5 zonas se seleccionaron 22 mujeres transgénero hasta lograr el número estimado de la muestra. Los criterios de inclusión fueron: Considerarse principalmente mujer transgénero, sexo masculino asignado al nacer, mayores de 18 años, residencia en Cali, sin importar el lugar de procedencia, y expresar su voluntariedad de participar del estudio mediante firma del consentimiento informado. El criterio de exclusión fue estar bajo el efecto del alcohol o sustancias psicoactivas al momento de la encuesta.

Para la recolección de los datos se utilizó una adaptación realizada por las autoras de la Encuesta de Acceso a Servicios de Salud para Hogares Colombianos -EASS, 2016- diseñada y validada en Colombia por Arrivillaga y colegas en 2016 (15). La encuesta original cuenta de 5 módulos así: perfil sociodemográfico, acceso a servicios preventivos, acceso a servicios curativos y gasto de bolsillo en acceso. Para este estudio se diseñó y validó por jueces y prueba piloto el módulo de acceso a la atención específica donde se evaluó el acceso a servicios de acuerdo con las necesidades de salud de las mujeres transgénero. La encuesta final, EASS-MUJERES TRANS, tuvo un total de 88 ítems.

La encuesta fue aplicada por personal previamente capacitado, entre los meses de agosto y noviembre del año 2015. Antes de la aplicación se realizó el procedimiento relacionado con el consentimiento informado. El tiempo de aplicación por encuesta fue en promedio de una hora, y al final de la recolección de la información cada participante recibió un kit de aseo a manera de compensación por el tiempo dedicado a la entrevista. 
Se efectuó un análisis univariado de la totalidad de las variables evaluadas. La información fue organizada y resumida a través de tablas de frecuencias, cálculo de medidas de tendencia central, variabilidad y dispersión de acuerdo con el nivel de medición de cada variable. Una interpretación conjunta de los resultados obtenidos permitió el desarrollo del análisis descriptivo de la información recolectada. El procesamiento estadístico se realizó utilizando como recurso el software SPSS, versión 20.

\section{Resultados}

\section{Características sociodemográficas}

Fueron encuestadas un total de 109 mujeres transgénero. El $91 \%$ se reconoció como mujer transgénero, la edad del $87 \%$ estuvo entre los 20 y los 44 años; el 53.2\% solo terminó algunos años de secundaria; el $51.8 \%$ estaba dedicada al trabajo sexual y el $29.5 \%$ a la peluquería; los ingresos económicos del $75.2 \%$ estuvo por debajo del salario mínimo legal vigente colombiano ( $\$ 644,350$ pesos colombianos). El $98.1 \%$ de las mujeres transgénero encuestadas reportó que vivía en estrato socioeconómico 1 a 3 (niveles bajo y mediobajo, según la clasificación colombiana).

El $71.6 \%$ de las encuestadas se encontraban afiliadas al sistema general de seguridad social en salud (SGSSS); el $61.5 \%$ estaba en el régimen subsidiado, el $9.2 \%$ en el régimen contributivo, de las cuales el $70 \%$ era cotizante y $30 \%$ beneficiaria; y el $0.9 \%$ en régimen especial. El 28.4\% de las mujeres transgénero no tenía afiliación a ningún régimen de salud, y cuando se les preguntó sobre las razones para ello, el $38.7 \%$ planteó no tener claro cuál era el procedimiento que debía realizar para afiliarse.

Sobre el diagnóstico previo de una o más enfermedades se encontró que el 26.6\% refirió haber sido diagnosticada previamente con una enfermedad así: 73.4\% VIH/SIDA, $6.7 \%$ gastritis crónica, y el $3.3 \%$ artritis crónica, colon irritable, hepatitis, hipertensión, diabetes y/o tuberculosis (Tabla 1).

Tabla 1. Características sociodemográficas, Acceso a servicios de salud de Mujeres Trans, Cali, Colombia, 2015, $(n=109)$.

\begin{tabular}{|c|c|c|c|c|}
\hline \multirow[b]{2}{*}{ Variable } & \multirow[b]{2}{*}{$\mathrm{n}$} & \multirow[b]{2}{*}{$\%$} & \multicolumn{2}{|c|}{ IC $(95 \%)$} \\
\hline & & & LI & LS \\
\hline \multicolumn{5}{|l|}{ Edad } \\
\hline 18 a 19 años & 12 & $11.00 \%$ & $7 \%$ & $29 \%$ \\
\hline 20 a 29 años & 55 & $50.40 \%$ & $37 \%$ & $64 \%$ \\
\hline 30 a 44 años & 32 & $29.30 \%$ & $14 \%$ & $45 \%$ \\
\hline Más 45 años & 10 & $9.10 \%$ & $9 \%$ & $27 \%$ \\
\hline \multicolumn{5}{|l|}{ Identidad de género } \\
\hline Mujer & 1 & $0.90 \%$ & $18 \%$ & $19 \%$ \\
\hline Mujer Trans & 100 & $91.70 \%$ & $86 \%$ & $97 \%$ \\
\hline Persona Trans & 3 & $2.80 \%$ & $16 \%$ & $21 \%$ \\
\hline Travesti & 1 & $0.90 \%$ & $18 \%$ & $19 \%$ \\
\hline Transformista & 4 & $3.70 \%$ & $15 \%$ & $22 \%$ \\
\hline \multicolumn{5}{|l|}{ Estado civil } \\
\hline Casada & 1 & $0.90 \%$ & $18 \%$ & $19 \%$ \\
\hline Unión libre & 11 & $10.10 \%$ & $8 \%$ & $28 \%$ \\
\hline Viuda & 3 & $2.80 \%$ & $16 \%$ & $21 \%$ \\
\hline Soltera & 92 & $84.40 \%$ & $77 \%$ & $92 \%$ \\
\hline NS/NR & 2 & $1.80 \%$ & $17 \%$ & $20 \%$ \\
\hline \multicolumn{5}{|l|}{ Etnia } \\
\hline Indígena & 4 & $3.60 \%$ & $15 \%$ & $22 \%$ \\
\hline Afrocolombiano & 26 & $23.90 \%$ & $8 \%$ & $40 \%$ \\
\hline Mestizo & 79 & $72.50 \%$ & $63 \%$ & $82 \%$ \\
\hline \multicolumn{5}{|l|}{ Nivel de educación } \\
\hline Algunos años de primaria & 10 & $9.21 \%$ & $9 \%$ & $27 \%$ \\
\hline Toda la primaria & 19 & $17.40 \%$ & $0 \%$ & $34 \%$ \\
\hline Algunos años de secundaria & 58 & $53.20 \%$ & $40 \%$ & $66 \%$ \\
\hline Toda la secundaria & 13 & $11.90 \%$ & $6 \%$ & $30 \%$ \\
\hline Técnica o tecnología & 6 & $5.50 \%$ & $13 \%$ & $24 \%$ \\
\hline Universidad completa & 2 & $180 \%$ & $17 \%$ & $20 \%$ \\
\hline Ninguno & 1 & $0.90 \%$ & $18 \%$ & $19 \%$ \\
\hline
\end{tabular}




\begin{tabular}{|c|c|c|c|c|}
\hline \multicolumn{5}{|l|}{ Ingresos } \\
\hline Menos de 1 SMMLV & 82 & $75.00 \%$ & $66 \%$ & $84 \%$ \\
\hline Entre 1 y 3,5 SMMLV & 27 & $25.00 \%$ & $9 \%$ & $41 \%$ \\
\hline \multicolumn{5}{|l|}{ Situación laboral } \\
\hline Peluquería, estilista & 41 & $29.50 \%$ & $16 \%$ & $43 \%$ \\
\hline Trabajo informal & 2 & $1.40 \%$ & $-15 \%$ & $18 \%$ \\
\hline Propietaria de pequeño negocio & 1 & $0.70 \%$ & $-16 \%$ & $17 \%$ \\
\hline Vinculación con empresa & 2 & $1.40 \%$ & $-15 \%$ & $18 \%$ \\
\hline Líder/lideresa comunitaria & 4 & $2.90 \%$ & $-14 \%$ & $19 \%$ \\
\hline Prostitución & 72 & $51.80 \%$ & $40 \%$ & $63 \%$ \\
\hline Striptease & 2 & $1.40 \%$ & $-15 \%$ & $18 \%$ \\
\hline Cyber show & 8 & $5.80 \%$ & $-10 \%$ & $22 \%$ \\
\hline Independiente & 7 & $5.10 \%$ & $-11 \%$ & $21 \%$ \\
\hline \multicolumn{5}{|l|}{ Estrato socioeconómico } \\
\hline Estrato 1 & 31 & $25.00 \%$ & $10 \%$ & $40 \%$ \\
\hline Estrato 2 & 46 & $44.00 \%$ & $30 \%$ & $58 \%$ \\
\hline Estrato 3 & 30 & $29.00 \%$ & $13 \%$ & $45 \%$ \\
\hline Estrato 4 & 1 & $1.00 \%$ & $-19 \%$ & $21 \%$ \\
\hline Estrato 5 & 1 & $1.00 \%$ & $-19 \%$ & $21 \%$ \\
\hline \multicolumn{5}{|l|}{ Tipo de vivienda } \\
\hline Propia pagada & 15 & $14.00 \%$ & $-4 \%$ & $32 \%$ \\
\hline Propia pagando & 8 & $6.00 \%$ & $-10 \%$ & $22 \%$ \\
\hline Arriendo & 52 & $48.00 \%$ & $34 \%$ & $62 \%$ \\
\hline Familiar & 2 & $2.00 \%$ & $-17 \%$ & $21 \%$ \\
\hline Pieza arriendo & 13 & $12.00 \%$ & $-6 \%$ & $30 \%$ \\
\hline Pieza por noche & 1 & $1.00 \%$ & $-19 \%$ & $21 \%$ \\
\hline En un cuarto de hotel & 18 & $17.00 \%$ & $0 \%$ & $34 \%$ \\
\hline \multicolumn{5}{|l|}{ Con quien vive } \\
\hline Sola & 40 & $37.00 \%$ & $22 \%$ & $52 \%$ \\
\hline Con su pareja & 11 & $10.00 \%$ & $-8 \%$ & $28 \%$ \\
\hline Con sus padres y hermanos & 24 & $22.00 \%$ & $5 \%$ & $39 \%$ \\
\hline Con sus padres & 19 & $17.00 \%$ & $0 \%$ & $34 \%$ \\
\hline Otro familiar & 5 & $5.00 \%$ & $-14 \%$ & $24 \%$ \\
\hline Con un amigo/a & 10 & $9.00 \%$ & $-9 \%$ & $27 \%$ \\
\hline \multicolumn{5}{|l|}{ Régimen de salud } \\
\hline Contributivo & 10 & $9.00 \%$ & $-9 \%$ & $27 \%$ \\
\hline Subsidiado & 67 & $61.00 \%$ & $49 \%$ & $73 \%$ \\
\hline No afiliado & 31 & $29.00 \%$ & $13 \%$ & $45 \%$ \\
\hline Especial & 1 & $1.00 \%$ & $-19 \%$ & $21 \%$ \\
\hline \multicolumn{5}{|l|}{ Tipo de afiliación } \\
\hline Cotizante & 7 & $70.00 \%$ & $36 \%$ & $104 \%$ \\
\hline Beneficiario & 3 & $30.00 \%$ & $-22 \%$ & $82 \%$ \\
\hline
\end{tabular}

${ }^{*}$ NS/NR: No sabe/No responde, SMMLV: Salario mínimo mensual legal vigente

\section{Acceso a servicios preventivos}

En este componente se describe el acceso a servicios preventivos por grupos de edad de acuerdo con la norma nacional, Resolución 412 de 2000. En términos generales, se encontró que existe una baja promoción de servicios de salud mental en el grupo más joven de 18 a 19 años; que la promoción y el uso de servicios se presentaron en valores similares entre los 20 y los 44 años, y que a medida que se incrementa la edad hubo mayor promoción de servicios por parte de las Entidades Promotoras de Salud (EPS) reportadas y mayores servicios utilizados. 
Al 25\% de las encuestadas entre 18 y 19 años de edad en el último año les han ofrecido consulta médica preventiva; solo al $8 \%$ consejería psicológica para trastornos de ansiedad, depresión y alimentación. En este grupo, el mayor uso fue por salud oral. En el grupo de 20 a 29 años, tanto la promoción por parte de las EPS como el uso de servicios mostraron valores similares. Aunque en un bajo porcentaje, el servicio más promovido fue el de la toma de tensión arterial con un $14 \%$. Se destaca que al $89 \%$ de las mujeres transgénero, las EPS no les ofrecieron servicios preventivos de pruebas de detección de Infecciones de Transmisión Sexual (ITS) y de VIH/SIDA que están contemplados en el Plan Obligatorio de Salud (POS), como tampoco se encontró mayor utilización.

En el grupo de 30 a 34 años la promoción de servicios preventivos y el uso fueron similares en cuanto a consulta médica, tensión arterial, toma de colesterol total y glicemia, con valores entre 34 y 50\%. La promoción del servicio de tacto rectal y antígeno prostático estuvo en niveles bajos de $12 \%$ y $9 \%$, respectivamente; solo un $9 \%$ de las encuestadas utilizó estos servicios.

En el grupo de mayores de 45 años se encontró mayor promoción de servicios preventivos por parte de las EPS, y también mayor utilización. Lo que más se promueve y se utiliza es toma de colesterol total y colesterol HDL, uroanálisis, triglicéridos, creatinina, glicemia y toma de tensión arterial. Se evidenció baja promoción de servicios preventivos de colonoscopias, y se elevó el uso de antígeno prostático y tacto rectal al 30\% y $40 \%$ (Tabla 2).

Tabla 2. Acceso a servicios preventivos por grupos de edad conforme a Resolución 412 de año 2000. Mujeres Trans, Cali, Colombia. 2015.

\begin{tabular}{|c|c|c|c|c|c|c|c|c|}
\hline \multirow{2}{*}{$\begin{array}{l}\text { Servicios preventivos } \\
\text { Por grupos de edad }\end{array}$} & \multicolumn{2}{|c|}{$\begin{array}{c}\text { Servicios } \\
\text { promovidos } \\
\text { por las EPS }\end{array}$} & \multicolumn{2}{|c|}{ IC $(95 \%)$} & \multicolumn{2}{|c|}{$\begin{array}{l}\text { Servicios } \\
\text { utilizados por } \\
\text { mujeres Trans }\end{array}$} & \multicolumn{2}{|c|}{ IC $(95 \%)$} \\
\hline & $\mathrm{n}$ & $\%$ & LI & LS & $\mathrm{n}$ & $\%$ & LI & LS \\
\hline \multicolumn{9}{|c|}{ De 18 a 19 años de vida $(n=12)$} \\
\hline Consulta médica preventiva & 3 & $25.00 \%$ & $-24 \%$ & $74 \%$ & 1 & $8.00 \%$ & $-45 \%$ & $61 \%$ \\
\hline Control por enfermería & 2 & $24.00 \%$ & $-35 \%$ & $83 \%$ & 0 & $0.00 \%$ & $0.00 \%$ & $0.00 \%$ \\
\hline $\begin{array}{l}\text { Consejería uso de drogas, } \\
\text { alcohol, tabaco }\end{array}$ & 2 & $24.00 \%$ & $-35 \%$ & $83 \%$ & 0 & $0.00 \%$ & $0.00 \%$ & $0.00 \%$ \\
\hline $\begin{array}{l}\text { Consulta médica o psicológica } \\
\text { por algún trastorno de } \\
\text { alimentación (anorexia, bulimia) }\end{array}$ & 1 & $8.00 \%$ & $-45 \%$ & $61 \%$ & 0 & $0.00 \%$ & $0.00 \%$ & $0.00 \%$ \\
\hline $\begin{array}{l}\text { Consulta médica o } \\
\text { psicológica por algún } \\
\text { trastorno como: depresión, } \\
\text { ansiedad, intento de suicidio }\end{array}$ & 1 & $8.00 \%$ & $-45 \%$ & $61 \%$ & 0 & $0.00 \%$ & $0.00 \%$ & $0.00 \%$ \\
\hline Valoración por agudeza visual & 1 & $8.00 \%$ & $-45 \%$ & $61 \%$ & 0 & $0.00 \%$ & $0.00 \%$ & $0.00 \%$ \\
\hline Consulta por salud oral (limpieza) & 2 & $24.00 \%$ & $-35 \%$ & $83 \%$ & 2 & $24.00 \%$ & $-35 \%$ & $83 \%$ \\
\hline Toma de presión arterial & 2 & $24.00 \%$ & $-35 \%$ & $83 \%$ & 1 & $8.00 \%$ & $-45 \%$ & $61 \%$ \\
\hline Toma de glicemia & 2 & $24.00 \%$ & $-35 \%$ & $83 \%$ & 0 & 0 & & \\
\hline \multicolumn{9}{|c|}{ De 20 a 29 años $(n=55)$} \\
\hline Consulta médica preventiva & 6 & $11.00 \%$ & $-14 \%$ & $36 \%$ & 6 & $11.00 \%$ & $-14 \%$ & $36 \%$ \\
\hline Toma de tensión arterial & 8 & $14.00 \%$ & $-10 \%$ & $38 \%$ & 7 & $13.00 \%$ & $-12 \%$ & $38 \%$ \\
\hline Prueba ELISA para VIH & 6 & $11.00 \%$ & $-14 \%$ & $36 \%$ & 7 & $13.00 \%$ & $-12 \%$ & $38 \%$ \\
\hline Prueba VDRL para sífilis & 6 & $11.00 \%$ & $-14 \%$ & $36 \%$ & 6 & $11.00 \%$ & $-14 \%$ & $36 \%$ \\
\hline \multicolumn{9}{|c|}{ De 30 a 44 años $(n=32)$} \\
\hline Consulta médica & 14 & $43.00 \%$ & $17 \%$ & $69 \%$ & 16 & $50.00 \%$ & $26 \%$ & $75 \%$ \\
\hline Toma de tensión arterial & 15 & $47.00 \%$ & $22 \%$ & $72 \%$ & 14 & $43.00 \%$ & $17 \%$ & $69 \%$ \\
\hline Toma de colesterol total & 11 & $34.00 \%$ & $6 \%$ & $62 \%$ & 12 & $37.00 \%$ & $10 \%$ & $64 \%$ \\
\hline Toma de glicemia & 12 & $37.00 \%$ & $10 \%$ & $64 \%$ & 12 & $37.00 \%$ & $10 \%$ & $64 \%$ \\
\hline Toma de tacto rectal & 4 & $12.00 \%$ & $-20 \%$ & $44 \%$ & 3 & $9.00 \%$ & $-23 \%$ & $41 \%$ \\
\hline Toma de antígeno prostático & 3 & $9.00 \%$ & $-23 \%$ & $41 \%$ & 3 & $9.00 \%$ & $-23 \%$ & $41 \%$ \\
\hline
\end{tabular}




\begin{tabular}{|c|c|c|c|c|c|c|c|c|}
\hline \multirow[b]{2}{*}{ Consulta médica del quinquenio } & & \multicolumn{7}{|c|}{ Mayores de 45 años $(n=10)$} \\
\hline & 4 & $40.00 \%$ & $-8 \%$ & $88 \%$ & 4 & $40.00 \%$ & $-8 \%$ & $88 \%$ \\
\hline Sangre oculta en heces & 2 & $20.00 \%$ & $-35 \%$ & $75 \%$ & 1 & $10.00 \%$ & $-49 \%$ & $69 \%$ \\
\hline Colonoscopia & 1 & $10.00 \%$ & $-49 \%$ & $69 \%$ & 0 & 0 & & \\
\hline Toma de tacto rectal & 4 & $40.00 \%$ & $-8 \%$ & $88 \%$ & 3 & $30.00 \%$ & $-22 \%$ & $82 \%$ \\
\hline Toma de antígeno prostático & 4 & $40.00 \%$ & $-8 \%$ & $88 \%$ & 4 & $40.00 \%$ & $-8 \%$ & $88 \%$ \\
\hline Densitometría ósea & 4 & $40.00 \%$ & $-8 \%$ & $88 \%$ & 3 & $30.00 \%$ & $-22 \%$ & $82 \%$ \\
\hline $\begin{array}{l}\text { Toma de colesterol total y } \\
\text { colesterol HDL }\end{array}$ & 6 & $60.00 \%$ & $21 \%$ & $99 \%$ & 5 & $50.00 \%$ & $6 \%$ & $94 \%$ \\
\hline Uroanálisis & 6 & $60.00 \%$ & $21 \%$ & $99 \%$ & 5 & $50.00 \%$ & $6 \%$ & $94 \%$ \\
\hline Triglicéridos & 6 & $60.00 \%$ & $21 \%$ & $99 \%$ & 5 & $50.00 \%$ & $6 \%$ & $94 \%$ \\
\hline Creatinina & 6 & $60.00 \%$ & $21 \%$ & $99 \%$ & 5 & $50.00 \%$ & $6 \%$ & $94 \%$ \\
\hline Glicemia & 6 & $60.00 \%$ & $21 \%$ & $99 \%$ & 5 & $50.00 \%$ & $6 \%$ & $94 \%$ \\
\hline Toma de presión arterial & 6 & $60.00 \%$ & $21 \%$ & $99 \%$ & 5 & $50.00 \%$ & $6 \%$ & $94 \%$ \\
\hline
\end{tabular}

\section{Acceso a servicios curativos}

En este componente se describe el acceso a los servicios curativos en cuanto a la utilización de los servicios de urgencias, consulta por médico general, especialista y hospitalización.

En acceso a servicios de urgencias se observó que las mujeres transgénero en un $23 \%$ requirieron atención; respecto al tiempo de espera entre el momento de llegar a urgencias y la atención de consulta médica, en un $32 \%$ fue inmediata, un 52\% esperó de 30 minutos a una hora. El 84\% manifestó que su problema de salud fue resuelto, aunque la calidad de la atención fue calificada mala y muy mala en un $32 \%$. Un $24 \%$ reportó haber costeado la atención con recursos propios $\mathrm{y} / \mathrm{o}$ familiares.

Por su parte, la consulta con médico general en los últimos 6 meses estuvo en un $18 \%$; el $60 \%$ fue remitido a especialista. Al 27\% la EPS no le entregó medicamentos y al 19\% solo algunos medicamentos; la principal razón para ello fue que no estaban incluidos en el POS. E1 25\% calificó la atención por médico general o especialista como mala o muy mala. Por el contrario, la calidad de la hospitalización fue valorada positivamente por un $88 \%$ de las encuestadas (Tabla 3).

Tabla 3. Acceso a servicios curativos. Mujeres Trans, Cali, Colombia. 2015

\begin{tabular}{|c|c|c|c|c|}
\hline & & & \multicolumn{2}{|c|}{ IC (95\%) } \\
\hline Servicios curativos & $\mathrm{n}$ & $\%$ & LI & LS \\
\hline \multicolumn{5}{|c|}{ En los últimos 6 meses, acudió a servicio de urgencias } \\
\hline No & 84 & $77.00 \%$ & 0.68 & 0.86 \\
\hline $\mathrm{Si}$ & 25 & $23.00 \%$ & 0.07 & 0.39 \\
\hline \multicolumn{5}{|c|}{ Tiempo entre el momento de llegar al servicio de urgencias y la consulta médica } \\
\hline Inmediatamente & 8 & $32.00 \%$ & 0.00 & 0.64 \\
\hline En máximo 30 minutos & 4 & $16.00 \%$ & -0.20 & 0.52 \\
\hline Entre 31 minutos y una hora & 9 & $36.00 \%$ & 0.05 & 0.67 \\
\hline Entre una hora y dos horas & 1 & $4.00 \%$ & -0.34 & 0.42 \\
\hline Más de dos horas & 3 & $12.00 \%$ & -0.25 & 0.49 \\
\hline \multicolumn{5}{|c|}{ Asistencia médica en el servicio de urgencias para solucionar el problema de salud } \\
\hline No & 3 & $12.00 \%$ & -0.25 & 0.49 \\
\hline $\mathrm{Si}$ & 21 & $84.00 \%$ & 0.68 & 1.00 \\
\hline NS/NR & 1 & $4.00 \%$ & -0.34 & 0.42 \\
\hline \multicolumn{5}{|c|}{ La calidad del servicio de urgencias fue } \\
\hline Muy buena & 3 & $12.00 \%$ & -0.25 & 0.49 \\
\hline Buena & 14 & $56.00 \%$ & 0.30 & 0.82 \\
\hline Mala & 6 & $24.00 \%$ & -0.10 & 0.58 \\
\hline Muy Mala & 2 & $8.00 \%$ & -0.30 & 0.46 \\
\hline
\end{tabular}




\begin{tabular}{lcccc}
\hline Fuentes utilizadas para cubrir los costos de atención en urgencias \\
EPS contributivo & 1 & $2.00 \%$ & -0.25 & 0.29 \\
EPS subsidiado & 18 & $72.00 \%$ & 0.51 & 0.93 \\
Recursos propios y/o familiares & 6 & $24.00 \%$ & -0.10 & 0.58 \\
EPS contributivo & 1 & $2.00 \%$ & -0.25 & 0.29
\end{tabular}

En los últimos 6 meses cuando ha tenido algún problema de salud, ha consultado a médico general

$\begin{array}{lcccc}\text { No } & 34 & 31.00 \% & 0.15 & 0.47 \\ \mathrm{Si} & 20 & 18.00 \% & 0.01 & 0.35 \\ \text { No he tenido problema de salud } & 54 & 50.00 \% & 0.37 & 0.63 \\ \text { NS/NR } & 1 & 1.00 \% & -0.19 & 0.21\end{array}$

Fuentes utilizadas para cubrir los costos de la consulta médica general

$\begin{array}{lcrrl}\text { EPS contributivo } & 1 & 5.00 \% & -0.38 & 0.48 \\ \text { EPS subsidiado } & 17 & 85.00 \% & 0.68 & 1.02 \\ \text { Recursos propios y/o familiares } & 1 & 5.00 \% & -0.38 & 0.48 \\ \text { Otro } & 1 & 5.00 \% & -0.38 & 0.48\end{array}$

Fue remitido a especialista por el médico general

$\begin{array}{lcccc}\text { No } & 8 & 40.00 \% & 0.06 & 0.74 \\ \mathrm{Si} & 12 & 60.00 \% & 0.32 & 0.88\end{array}$

Especialista al que fue remitido

Infectólogo

$12 \quad 60.00 \%$

0.32

0.88

Internista

$\begin{array}{llll}1 & 8.00 \% & -0.45 & 0.61\end{array}$

Cirugía

$25.00 \%$

$-0.24$

0.74

Dermatólogo

$17.00 \%$

$-0.35$

0.69

Psiquiatría

$17.00 \%$

$-0.35$

0.69

Urólogo

$8.00 \%$

$-0.45$

0.61

Traumatólogo

$8.00 \%$

$-0.45$

0.61

Neumólogo

$8.00 \%$

$-0.45$

0.61

Ortopedista

$8.00 \%$

$-0.45$

0.61

Endocrinólogo

$8.00 \%$

$-0.45$

0.61

Gastroenterólogo

$8.00 \%$

$-0.45$

0.61

Fuente utilizada para cubrir los costos de atención por especialista

EPS contributivo

$1 \quad 8.00 \% \quad-0.45$

0.61

EPS subsidiado

$10 \quad 84.00 \%$

0.61

0.61

No fue atendido

$1 \quad 8.00 \%$

$-0.45$

1.07

Por la situación consultada, le formularon medicamentos

$\begin{array}{lllll}\text { No } & 1 & 8.00 \% & -0.45 & 0.61\end{array}$

$\begin{array}{lllll}\mathrm{Si} & 11 & 92.00 \% & 0.76 & 1.08\end{array}$

Estos medicamentos fueron entregados por la EPS

$\begin{array}{lllll}\text { No } & 3 & 27.00 \% & -0.23 & 0.77 \\ \text { Sí, todos } & 6 & 54.00 \% & 0.14 & 0.94 \\ \text { Si, algunos } & 2 & 19.00 \% & -0.35 & 0.73\end{array}$

Razón por la que no fueron entregados los medicamentos

$\begin{array}{lllll}\text { No estaban incluidos en el POS } & 2 & 67.00 \% & 0.02 & 1.32\end{array}$

$\begin{array}{lllll}\text { Acudió a médico particular } & 1 & 33.00 \% & -0.59 & 1.25\end{array}$

La calidad de la prestación del servicio de salud medicina general o especializada fue

\begin{tabular}{lcccc} 
Muy buena & 3 & $15.00 \%$ & -0.25 & 0.55 \\
Buena & 12 & $60.00 \%$ & 0.32 & 0.88 \\
Mala & 4 & $20.00 \%$ & -0.19 & 0.59 \\
Muy Mala & 1 & $5.00 \%$ & -0.38 & 0.48 \\
\hline
\end{tabular}




\begin{tabular}{|c|c|c|c|c|}
\hline \multicolumn{5}{|c|}{ En los últimos 6 meses, ha tenido alguna hospitalización } \\
\hline No & 94 & $92.00 \%$ & 0.87 & 0.97 \\
\hline $\mathrm{Si}$ & 8 & $8.00 \%$ & -0.11 & 0.27 \\
\hline \multicolumn{5}{|c|}{ La hospitalización fue } \\
\hline Por urgencias & 7 & $88.00 \%$ & 0.64 & 1.12 \\
\hline Programada & 1 & $12.00 \%$ & -0.52 & 0.76 \\
\hline \multicolumn{5}{|c|}{$\begin{array}{l}\text { Durante la hospitalización le brindaron la asistencia médica necesaria para solucionar } \\
\text { el problema de salud }\end{array}$} \\
\hline No & 1 & $12.00 \%$ & -0.52 & 0.76 \\
\hline $\mathrm{Si}$ & 7 & $88.00 \%$ & 0.64 & 1.12 \\
\hline \multicolumn{5}{|c|}{ La calidad de la hospitalización fue } \\
\hline Muy Buena & 2 & $25.00 \%$ & -0.35 & 0.85 \\
\hline Buena & 5 & $63.00 \%$ & 0.21 & 1.05 \\
\hline Mala & 1 & $12.00 \%$ & -0.52 & 0.76 \\
\hline
\end{tabular}

*EPS: Empresa promotora de salud, POS: Plan obligatorio de salud.

\section{Acceso a servicios específicos para atender necesidades de mujeres transgénero}

Se encontró que un $67.5 \%$ manifestó haber tenido problemas de salud en el último año, siendo el dolor de cabeza, la fiebre, el dolor de espalda, los problemas digestivos, las condiciones de salud que más las afectan. Un $25 \%$ de los entrevistados buscó atención acudiendo a farmacias y droguerías, por el contrario, el $61 \%$ buscó atención en centros de salud, puestos de salud y hospitales, de los cuales solo un $48 \%$ recibió tratamiento médico.

Para el proceso de transición corporal se indagó por el uso de hormonas, y se identificó que un $91 \%$ las utilizan, pero solo un $6 \%$ recibe atención médica para su uso. El 98\% lo hace por fuera de los servicios de salud. Sobre el uso de sustancias inyectables, el $70 \%$ se realizó algún procedimiento estético, siendo la silicona fluida la sustancia más utilizada, seguida de aceites vegetales. La administración de estas sustancias se dio en un $40.3 \%$ por medio de una esteticista, $23.4 \%$ con amigos y amigas, y un $5.2 \%$ auto administrada. Con respecto al acceso a cirugías plásticas el 37\% alguna vez se realizó una cirugía estética, el 93\% de este grupo lo hizo en clínicas y centros especializados de cirugía plástica.

Respecto al acceso al condón, el $89.7 \%$ reportó adquirirlo en las organizaciones no gubernamentales, solo un $2.8 \%$ lo obtuvo del sistema de salud. Sobre el acceso a la atención por psicología, el $72.4 \%$ indicó no tener necesidad de este servicio, el 17.4\% manifestó haber accedido por depresión y $4.4 \%$ por ansiedad (Tabla 4 ).

Tabla 4. Acceso a servicios específicos. Mujeres Trans, Cali, Colombia. 2015

\begin{tabular}{|c|c|c|c|c|}
\hline \multirow[b]{2}{*}{ Servicios específicos para mujeres Trans } & \multirow[b]{2}{*}{$\mathrm{n}$} & \multirow[b]{2}{*}{$\%$} & \multicolumn{2}{|c|}{ IC (95\%) } \\
\hline & & & LI & LS \\
\hline \multicolumn{5}{|c|}{ En los últimos 12 meses ha tenido molestias o problemas de salud } \\
\hline No & 45 & $49.00 \%$ & $34 \%$ & $64 \%$ \\
\hline $\mathrm{Si}$ & 62 & $68.00 \%$ & $56 \%$ & $80 \%$ \\
\hline NS/NR & 2 & $2.00 \%$ & $-17 \%$ & $21 \%$ \\
\hline \multicolumn{5}{|l|}{ Qué tipo de molestias ha presentado } \\
\hline Respiratorias & 10 & $16.00 \%$ & $-7 \%$ & $39 \%$ \\
\hline Dolor de cabeza & 22 & $35.00 \%$ & $15 \%$ & $55 \%$ \\
\hline Fiebre & 17 & $27.00 \%$ & $6 \%$ & $48 \%$ \\
\hline Falta de energía, cansancio & 8 & $13.00 \%$ & $-10 \%$ & $36 \%$ \\
\hline De espalda & 17 & $27.00 \%$ & $6 \%$ & $48 \%$ \\
\hline De la visión & 1 & $2.00 \%$ & $-25 \%$ & $29 \%$ \\
\hline Del oído & 3 & $5.00 \%$ & $-20 \%$ & $30 \%$ \\
\hline Problemas digestivos & 10 & $16.00 \%$ & $-7 \%$ & $39 \%$ \\
\hline De la piel & 4 & $6.00 \%$ & $-17 \%$ & $29 \%$ \\
\hline Desmayos & 3 & $5.00 \%$ & $-20 \%$ & $30 \%$ \\
\hline Alergias & 3 & $5.00 \%$ & $-20 \%$ & $30 \%$ \\
\hline
\end{tabular}




\begin{tabular}{|c|c|c|c|c|}
\hline Musculares & 7 & $11.00 \%$ & $-12 \%$ & $34 \%$ \\
\hline Resfriados & 3 & $5.00 \%$ & $-20 \%$ & $30 \%$ \\
\hline Molestias para orinar & 7 & $11.00 \%$ & $-12 \%$ & $34 \%$ \\
\hline Molestias en el área del ano & 1 & $2.00 \%$ & $-25 \%$ & $29 \%$ \\
\hline Secreción genital & 1 & $2.00 \%$ & $-25 \%$ & $29 \%$ \\
\hline Úlceras, llagas, granos en zona genital o anal & 2 & $3.00 \%$ & $-21 \%$ & $27 \%$ \\
\hline Ninguna de las anteriores & 2 & $3.00 \%$ & $-21 \%$ & $27 \%$ \\
\hline Otra & 15 & $24.00 \%$ & $2 \%$ & $46 \%$ \\
\hline \multicolumn{5}{|c|}{ Para estos problemas o molestias que tuvo, recibió tratamiento médico } \\
\hline No & 32 & $51.00 \%$ & $34 \%$ & $68 \%$ \\
\hline $\mathrm{Si}$ & 29 & $48.00 \%$ & $30 \%$ & $66 \%$ \\
\hline NS/NR & 1 & $2.00 \%$ & $-25 \%$ & $29 \%$ \\
\hline \multicolumn{5}{|c|}{ A qué sitio acude generalmente cuando tiene problemas de salud } \\
\hline Centro puesto de salud, hospital & 34 & $54.00 \%$ & $37 \%$ & $71 \%$ \\
\hline Clínica privada & 2 & $3.00 \%$ & $-21 \%$ & $27 \%$ \\
\hline Consultorio médico particular & 3 & $5.00 \%$ & $-20 \%$ & $30 \%$ \\
\hline En el hogar o ayuda familiar (remedio casero) & 1 & $2.00 \%$ & $-25 \%$ & $29 \%$ \\
\hline Farmacia & 19 & $31.00 \%$ & $10 \%$ & $52 \%$ \\
\hline A ningún sitio & 3 & $5.00 \%$ & $-20 \%$ & $30 \%$ \\
\hline \multicolumn{5}{|l|}{ Ha usado o está usando hormonas } \\
\hline No & 10 & $9.00 \%$ & $-9 \%$ & $27 \%$ \\
\hline $\mathrm{Si}$ & 99 & $91.00 \%$ & $85 \%$ & $97 \%$ \\
\hline \multicolumn{5}{|l|}{ Recibe o recibió atención médica para el uso } \\
\hline No & 88 & $94.00 \%$ & $89 \%$ & $99 \%$ \\
\hline $\mathrm{Si}$ & 6 & $6.00 \%$ & $-13 \%$ & $25 \%$ \\
\hline \multicolumn{5}{|l|}{ Quién le administra o administró las hormonas } \\
\hline Médico en el centro de salud, puesto de salud, hospital & 2 & $2.00 \%$ & $-17 \%$ & $21 \%$ \\
\hline Enfermera & 2 & $2.00 \%$ & $-17 \%$ & $21 \%$ \\
\hline Médico consultorio particular & 2 & $2.00 \%$ & $-17 \%$ & $21 \%$ \\
\hline Peluquería, salón de belleza o estética & 6 & $6.00 \%$ & $-13 \%$ & $25 \%$ \\
\hline Con amigos 0 amigas / conocidos/as & 3 & $3.00 \%$ & $-16 \%$ & $22 \%$ \\
\hline Droguería & 49 & $50.00 \%$ & $36 \%$ & $64 \%$ \\
\hline Auto administrada & 35 & $35.00 \%$ & $19 \%$ & $51 \%$ \\
\hline \multicolumn{5}{|l|}{ Se ha inyectado alguna sustancia } \\
\hline No & 33 & $30.00 \%$ & $14 \%$ & $46 \%$ \\
\hline $\mathrm{Si}$ & 76 & $70.00 \%$ & $60 \%$ & $80 \%$ \\
\hline \multicolumn{5}{|c|}{ Qué sustancia o sustancias se ha inyectado en los últimos 12 meses } \\
\hline Aceites vegetales & 10 & $13.00 \%$ & $-8 \%$ & $34 \%$ \\
\hline Botox & 4 & $5.00 \%$ & $-16 \%$ & $26 \%$ \\
\hline Colágeno & 1 & $1.00 \%$ & $-19 \%$ & $21 \%$ \\
\hline Ácido hialurónico & 2 & $3.00 \%$ & $-21 \%$ & $27 \%$ \\
\hline Silicona Industrial & 3 & $4.00 \%$ & $-18 \%$ & $26 \%$ \\
\hline Silicona fluida & 70 & $92.00 \%$ & $86 \%$ & $98 \%$ \\
\hline Biopolímero & 2 & $3.00 \%$ & $-21 \%$ & $27 \%$ \\
\hline \multicolumn{5}{|l|}{ Quién le administró la sustancia } \\
\hline Enfermera & 4 & $5.00 \%$ & $-16 \%$ & $26 \%$ \\
\hline Médico consultorio particular & 5 & $7.00 \%$ & $-15 \%$ & $29 \%$ \\
\hline Peluquería, salón de belleza o estética & 8 & $10.00 \%$ & $-11 \%$ & $31 \%$ \\
\hline Con amigos o amigas / conocidos/as & 18 & $23.00 \%$ & $4 \%$ & $42 \%$ \\
\hline Esteticista & 31 & $41.00 \%$ & $24 \%$ & $58 \%$ \\
\hline
\end{tabular}




\begin{tabular}{|c|c|c|c|c|}
\hline Farmaceuta & 5 & $7.00 \%$ & $-15 \%$ & $29 \%$ \\
\hline Auto administrada & 5 & $7.00 \%$ & $-15 \%$ & $29 \%$ \\
\hline \multicolumn{5}{|c|}{ Usted se ha realizado una cirugía como parte de su transición corporal } \\
\hline Cirugía en los senos (mamoplastia) & 28 & $26.00 \%$ & $10 \%$ & $42 \%$ \\
\hline Cirugía de la nariz (rinoplastia) & 7 & $6.00 \%$ & $-12 \%$ & $24 \%$ \\
\hline Cirugía de glúteos (glutinoplastia) & 3 & $3.00 \%$ & $-16 \%$ & $22 \%$ \\
\hline Cirugía liposucción o lipectomía & 2 & $2.00 \%$ & $-17 \%$ & $21 \%$ \\
\hline No ha hecho ningún procedimiento & 69 & $63.00 \%$ & $52 \%$ & $74 \%$ \\
\hline \multicolumn{5}{|c|}{ En que sitios se realizó o se realiza estos procedimientos } \\
\hline Clínica o centro de cirugía plástica & 37 & $93.00 \%$ & $85 \%$ & $101 \%$ \\
\hline Estéticas, peluquería & 2 & $5.00 \%$ & $-25 \%$ & $35 \%$ \\
\hline Casa & 1 & $2.00 \%$ & $-25 \%$ & $29 \%$ \\
\hline \multicolumn{5}{|l|}{ En qué lugares adquiere los condones } \\
\hline Farmacia & 5 & $5.00 \%$ & $-14 \%$ & $24 \%$ \\
\hline Centro o puesto de salud & 3 & $3.00 \%$ & $-16 \%$ & $22 \%$ \\
\hline Fundaciones & 1 & $0.90 \%$ & $-18 \%$ & $19 \%$ \\
\hline Peluquerías o salón de belleza & 1 & $0.90 \%$ & $-18 \%$ & $19 \%$ \\
\hline ONG & 96 & $90.00 \%$ & $84 \%$ & $96 \%$ \\
\hline \multicolumn{5}{|c|}{ Ha tenido la necesidad de acudir a una consulta psicológica en los últimos 6 meses por } \\
\hline Problemas de comportamiento & 2 & $2.18 \%$ & $-18 \%$ & $22 \%$ \\
\hline Tendencias suicidas & 1 & $0.90 \%$ & $-18 \%$ & $19 \%$ \\
\hline Depresión & 19 & $17.00 \%$ & $0 \%$ & $34 \%$ \\
\hline Ansiedad & 5 & $4.50 \%$ & $-14 \%$ & $23 \%$ \\
\hline Consumo SPA & 1 & $0.90 \%$ & $-18 \%$ & $19 \%$ \\
\hline No he tenido la necesidad & 79 & $72.00 \%$ & $62 \%$ & $82 \%$ \\
\hline Otra & 2 & $2.00 \%$ & $-17 \%$ & $21 \%$ \\
\hline \multicolumn{5}{|l|}{ Estigma y discriminación } \\
\hline $\begin{array}{l}\text { Ha sentido discriminación del personal de salud } \\
\text { por su género }\end{array}$ & 7 & $6.00 \%$ & $-12 \%$ & $24 \%$ \\
\hline
\end{tabular}

*NS/NR: No sabe/No responde, ONG: Organización no gubernamental, SPA: Sustancias psicoactivas.

\section{Gasto de bolsillo en acceso a servicios de salud}

Este componente evaluó el gasto de bolsillo en acceso a servicios de salud, encontrando que el copago y la cuota moderada pagada por la atención recibida estuvieron entre $\$ 2,100$ a $\$ 24,000$ COP. Con respecto a medicamentos, exámenes, procedimientos no autorizados o no pagados por la EPS el costo osciló entre $\$ 10,000$ y $\$ 80,000$ COP. Con respecto a otros gastos como procedimientos, transporte, alimentación, alojamiento el valor estuvo entre $\$ 2,100$ y $\$ 4,300.000$ COP.

\section{Discusión}

Este estudio tuvo como objetivo describir y analizar el acceso a los servicios de salud preventivos, curativos, de atención específica y gasto de bolsillo en mujeres transgénero en la ciudad de Cali, Colombia. Los hallazgos centrales mostraron que, si bien el $71.6 \%$ de las mujeres transgénero se encuentran cubiertas al SGSSS en Colombia, esto no garantiza el acceso oportuno a servicios preventivos, curativos y a la atención específica de acuerdo con sus necesidades. Como se ha planteado previamente, para interpretar este resultado es necesario tener en cuenta la diferencia entre los conceptos de cobertura y acceso, pues aun cuando una persona esté asegurada por el SGSSS, persisten barreras que pueden evitar que reciba atención en caso de necesitarla (16).

De otro lado, la Constitución Colombiana, en su artículo 16 establece que todas las personas tienen derecho al libre desarrollo de su personalidad sin más limitaciones que las que imponen los derechos de los demás y el orden jurídico. La garantía del derecho a la salud de las mujeres transgénero 
en su condición de ciudadanas hace que el reto para los servicios de salud sea una necesidad, pero también una obligación. No obstante, los resultados de este estudio mostraron la debilidad institucional por parte de las EPS para promover servicios preventivos y para prestar atención integral diferencial de acuerdo con sus necesidades. Por su identidad de género, las mujeres transgénero enfrentan una serie de situaciones de exclusión y la marginación general (17), que a su vez se expresan en los servicios salud. Así, el ejercicio de su ciudadanía en salud resulta amenazado dada la falta de atención, rechazo, estigma y discriminación.

Los hallazgos de este estudio son coherentes con la estructura del sistema de salud colombiano que está diseñado para invertir los recursos en la atención de la enfermedad y no en la prevención (18). Las instituciones siguen manteniendo modelos habituales de asistencia en salud, sin tener en cuenta las heterogeneidades y necesidades específicas de la población vulnerable; por su parte, las aseguradoras no garantizan el desarrollo de planes y acciones preventivas no contempladas en el POS (19). Asuntos como la prevención de infecciones de transmisión sexual y VIH, el consumo de sustancias psicoactivas y consejería para el fortalecimiento de la identidad de género no son atendidos. De esta manera, la debilidad en la prevención impone mayores riesgos de enfermar y morir en las mujeres transgénero que en el resto de la población general (20).

En cuanto al acceso a servicios curativos este estudio encontró que las mujeres transgénero enfrentan menos barreras en consulta externa, urgencias y hospitalización, en comparación con el acceso a servicios preventivos. En general, la percepción de estos servicios es de buena calidad, oportunidad y satisfacción en la atención de la necesidad. No obstante, en el acceso a consulta médica especializada se encontraron demoras de oportunidad y en el acceso a medicamentos se reportaron dificultades para obtenerlos, en especial, los no contemplados en el POS.

Con respecto al gasto de bolsillo, los ingresos económicos se relacionan con el acceso. En el caso de las mujeres transgénero entrevistadas, el $75.2 \%$ reportó ingresos por debajo del salario mínimo legal vigente colombiano, cuyo gasto se prioriza en satisfacción de necesidades de subsistencia como la alimentación y la vivienda, por encima de la atención en salud. Así, las condiciones de vida se convierten en una barrera para el acceso en general, de los servicios no cubiertos por el POS y para la atención de necesidades específicas relacionadas con su identidad género.

Con respecto al acceso a servicios específicos existe un determinante fundamental que se debe considerar en la atención y que se refiere a la identidad de género de las mujeres transgénero. Ellas luchan por reivindicar la identidad femenina que implica un tránsito de hombre a mujer, y para lo cual requieren una serie de procedimientos médicos, consejería psicológica y apoyo social que les permita alcanzar ese ideal de identidad. En el actual SGSSS colombiano tales procedimientos no están contemplados en el POS. Estos son: Concentraciones hormonales, procedimientos quirúrgicos de reasignación de sexo y acompañamiento psicosocial. Toda esta atención está prevista en protocolos de atención a nivel internacional en países como Estados Unidos, España, Chile y Argentina. Por el contrario, los resultados de este estudio evidencian que en Colombia no cuentan con acceso para la atención específica, y que las mujeres transgénero recurren a alternativas por fuera del sistema, que ponen en riesgo su salud y su vida. De hecho, diferentes estudios han encontrado, por ejemplo, que existen una serie de enfermedades asociadas al uso no supervisado de hormonas entre las cuales se encuentran la trombosis venosa profunda, trombo embolismo pulmonar (21-23), infarto al miocardio (13), diabetes (24), anemia, depresión (25) y daño hepático (26-28).

Los resultados de este estudio representan una alerta al proceso de implementación de la Política de Atención Integral en Salud (29) establecida en el Decreto 429 de 2016 en Colombia, Las acciones de atención a las mujeres transgénero requiere por parte de los entes rectores de salud, estrategias de formación para el personal de salud donde se incorpore la atención diferencial en salud con enfoque en construcciones de género e identidades diversas. Asimismo, la construcción de protocolos de atención médica y psicosocial con el fin de avanzar hacia real garantía del derecho a la salud de las mujeres transgénero en su condición de ciudadanas protegidas constitucionalmente para el libre desarrollo de su personalidad.

Como limitaciones, este estudio contó con la dificultad de realizar triangulación de información empírica dada la ausencia de otras investigaciones en acceso a los servicios de salud para mujeres transgénero en Colombia y en América Latina. De igual manera, el desarrollarse como un estudio observacional no permitió profundizar asuntos de la construcción de la identidad de género de las mujeres transgénero y su relación con los servicios de salud. Por esto, se recomiendan estudios cualitativos posteriores que permitan una mejor comprensión del fenómeno. En términos del análisis de datos, el tamaño de la muestra y su desagregación en grupos de edad en el módulo de acceso a servicios preventivos, impidió realizar análisis entre las variables del estudio. Es recomendable que estudios posteriores de tipo cuantitativo amplíen los tamaños muestrales de forma que puedan establecerse inferencias estadísticas potentes. Se sugiere la aplicación de la EASSMUJERES TRANS en otras ciudades en Colombia para identificar qué diferencias y similitudes existen en las condiciones de acceso los servicios de salud.

Por último, sería importante que las Direcciones Territoriales de Salud en Colombia, retomaran la metodología de intervención de actividades preventivas de proyectos como el del Fondo Mundial de VIH/SIDA con 
mujeres transgénero (30), e incorporen a los equipos actuales de atención primaria en salud el tema. Así se podrán desarrollar abordajes integrales con esta población, con los profesionales y con los gestores comunitarios de salud, tal como lo propone el Modelo Integral de Atención en Salud.

\section{Conclusiones}

Se requiere implementar la Política de Atención Integral en Salud establecida en el Decreto 429 de 2016 en Colombia con cambios sustanciales en los servicios de salud en relación a los grupos poblacionales diversos como las mujeres transgénero. Es necesario fortalecer el acceso a los servicios de salud tanto preventivos como curativos y, dada la precariedad de sus condiciones de vida, atenuar los efectos en el gasto de bolsillo en acceso mediante la facilitación del acceso a procedimientos requeridos por esta población. El acceso a servicios específicos requiere también establecer protocolos de atención diferencial dado los riesgos para la salud y la vida en los que las mujeres están incurriendo por falta de atención médica y psicosocial. Solo de esta manera se garantiza el pleno derecho a la salud y el respeto por el libre desarrollo de la personalidad establecido constitucionalmente en el país.

\section{Responsabilidades éticas}

Protección de personas y animales. Los autores declaran que en esta investigación no se han realizado experimentos en seres humanos ni en animales.

Confidencialidad de los datos. Los autores declaran que han seguido los protocolos de su centro de trabajo sobre la publicación de datos de pacientes y que todos los pacientes incluidos en el estudio han recibido información suficiente y han dado su consentimiento informado por escrito para participación en dicho estudio.

Derecho a la privacidad y consentimiento informado. Los autores declaran que han obtenido el consentimiento informado de los pacientes y/o sujetos referidos en el artículo. Este documento obra en poder del autor de correspondencia.

\section{Conflicto de intereses}

Los autores declaran no tener conflicto de interés.

\section{References}

1. República de Colombia. Ley 100 de 1993. [Internet]. 1993. [citado 20 marzo 2016]. Disponible en: https://docs.supersalud.gov.co/PortalWeb/Juridica/Leyes/ L0100_93.pdf
2. Tovar L, Arrivillaga M. Estado del arte de la investigación en acceso a los servicios de salud en Colombia, 20002013: revisión sistemática crítica. Rev. Gerenc. Polít. Salud. 2014; 13(27):17-26. Disponible en: https://doi.org/ 10.11144/Javeriana.rgyps13-27.eaia

3. Vargas J, Molina G. Acceso a los servicios de salud en seis ciudades de Colombia: limitaciones y consecuencias. Rev Fac Nac Salud Pública. 2009; 27(2): 121-130.

4. Baral S, Poteat T, Strömdahl S, Wirtz A, Guadamuz T, Beyrer C. Worldwide burden of HIV in transgender women: a systematic review and meta-analysis. Lancet Infect Dis. 2013; 13(3): 214-22. Disponible en: https://doi.org/10.1016/S1473-3099(12)70315-8

5. Bockting W. From construction to context: gender through the eyes of the transgendered. Siecus Rep. 1999; 28(1): 3-7.

6. Chárriez M. La transexualidad: ¿construcción de una identidad? Rev. Griot. 2013; 6(1): 18-28.

7. Bockting W, Miner M, Swinburne R, Hamilton A, Coleman E. Stigma, Mental Health, and Resilience in an Online Sample of the US Transgender Population. Am J Public Health. 2013; 103(5): 943-951. Disponible en: https://doi.org/10.2105/AJPH.2013.301241

8. Organización Panamericana de la Salud. Por la salud de las personas trans: Elementos para el desarrollo de la atención integral de personas trans y sus comunidades en Latinoamérica y el Caribe. [Internet]. 2012. [citado 20 marzo 2016]. Disponible en: http://www.paho.org/arg/ images/gallery/Blueprint $\% 20$ Trans $\% 20$ Espa\% C3\%83\% C2\%B1ol.pdf

9. Weeks J. The Languages of Sexuality. Routledge. London and New York. $1^{\text {a }}$ edición. Oxon. 2011.

10. Mecanismo de Coordinación de País. Resultados del estudio comportamiento sexual y prevalencia de infección por VIH en mujeres trans, en cuatro ciudades de Colombia. [Internet]. 2013. [citado 20 marzo 2016]. Disponible en: http://www.sidastudi.org/es/registro/ ff8081814223340201436d4f97be042f

11. Becerra A, de Luis Román D, Piedrola G. Morbilidad en pacientes transexuales con autotratamiento hormonal para cambio de sexo. Medicina Clínica. 1999; 113(13): 484-487.

12. García A. Tacones, Siliconas, Hormonas: Teoría Feminista y experiencias trans en Bogotá. [internet]. 2010. Universidad Nacional. [citado 20 marzo 2016]. Disponible e http://www.bdigital.unal.edu.co/2978/1/489177.2010.pdf

13. Elamin M, García M, Murad M, Erwin P, Montori V. Effect of sex steroid use on cardiovascular risk in transsexual individuals: a systematic review and meta-analyses. Clin Endocrinol. 2010; 72(1): 1-10. Disponible en: https://doi.org/10.1111/j.1365-2265.2009.03632.x

14. Organización Panamericana de la Salud (OPS). Metodología de triangulación para el análisis del tamaño poblacional y las brechas en la respuesta nacional en VIH orientada a la población transgénero. En reunión CONASIDA; 2015, febrero 17; Bogotá, Colombia: 1-40.

15. Arrivillaga M, Aristizábal J, Pérez M, Estrada V. Encuesta de acceso a servicios de salud para hogares colombianos. Gac Sanit. 2016; 30(6):415-420. Disponible en: https://doi.org/10.1016/j.gaceta.2016.05.008

16. Ayala J. La salud en Colombia: más cobertura, pero menos acceso. Documentos de Trabajo Sobre Economía Regional. Cartagena: Banco de la República. [internet]. 2014. [citado 20 marzo 2016]. Disponible en: 
http://www.banrep.gov.co/sites/default/files/publicaciones /archivos/dtser_204.pdf

17. Buriticá I. Las políticas públicas mujer y géneros y LGBT y el caso de las transgeneristas. La manzana de la discordia. 2010;(5)1: 35-43.

18. Vélez M. La salud en Colombia: Pasado, presente y futuro de un sistema en crisis. Editorial Penguin Random House. 2016.

19. Mogollón A, Vásquez M. Factores que inciden en el acceso a población desplazada a las instituciones prestadoras de servicios de salud en Colombia. Cad. Saude Pública. 2008; 24(4):745-754.

20. Clements-Nolle K, Marx R, Guzman R, Katz M. HIV prevalence, risk behaviors, health care use, and mental health status of transgender persons: implications for public health intervention. Am J Public Health. 2001; 91(6): 915-921.

21. Healthy People. Companion Document for Lesbian, Gay, Bisexual, and Transgender (LGBT) Health. [Internet]. 2010. [citado 20 marzo 2016]. Disponible en: http://www.nalgap.org/PDF/Resources/HP2010CDLGB THealth.pdf

22. Wiessing $L$, van Roosmalen $M$, Koedijk $P$, Bieleman $B$, Houweling $\mathrm{H}$. Silicones, hormones and HIV in transgender street prostitutes. AIDS. 1999; 13(16):2315-2316.

23. Shatzel J, Connelly K, DeLoughery T. Thrombotic issues in transgender medicine: A review. Am J Hematol. 2017; $92(2)$ : $204-208$. D i s p o n ible en: https://doi.org/10.1002/ajh.24593

24. Defreyne J, De Bacquer D, Shadid S, Lapauw B, T'Sjoen G. Is Type 1 Diabetes Mellitus More Prevalent Than Expected in Transgender Persons? A Local Observation. Sex Med. 2017; 5(3): 215-218. Disponible en: https://doi.org/10.1016/j.esxm.2017.06.004
25. Asscheman H, T'Sjoen G, Gooren L. Morbidity in crosssex hormone-treated transgender people. Joint meeting of the International Society of Endocrinology and the Endocrine Society ICE/ENDO. 2014.

26. Van Kesteren P, Asscheman H, Megens J, Gooren L. Mortality and morbidity in transsexual subjects treated with cross-sex hormones. Clin Endocrinol. 1997; 47(3): 337-342. Disponible en: https://doi.org/10.1046/j.13652265.1997.2601068.x

27. Hage J, Kanhai R, Oen A, van Diest P, Karim R. The devastating outcome of massive subcutaneous injection of highly viscous fluids in male-to-female transsexuals. Plast Reconstr Surg. 2001; 107(3): 734-741. Disponible en: https://doi.org/10.1097/00006534-20010300000013

28. Feldman J, Brown G, Deutsch M, Hembree W, Meyer W, Meyer-Bahlburg $\mathrm{H}$, et al. Priorities for Transgender Medical and Healthcare Research. Curr Opin Endocrinol Diabetes Obes. 2016; 23(2): 180-187. Disponible en: https://doi.org/10.1097/MED.0000000000000231

29. Ministerio de Salud y Protección Social. Política de Atención Integral en Salud. [internet]. 2016. [citado 20 marzo 2016]. Disponible en: https://www.minsalud.gov.co/ sites/rid/Lists/BibliotecaDigital/RIDE/DE/modelo-pais2016.pdf

30. Mecanismo de Coordinación de País. Guías rápidas de atención en VIH, con enfoque diferencial y de vulnerabilidad. Bogotá. [Internet]. 2015. [citado 20 marzo 2016]. Disponible en: old.integracionsocial.gov.co/ anexos/documentos/2015_centro_documentacion/1112 2015 Guia\%2520de\%2520consulta\%2520para\%2520I a\%2520atencion\%2520diferencial\%2520a\%2520perso nas $\% 252$ LBGTI\%2520en\%2520comisarias\%2520de $\% 2520$ familia $\% 2520$ en $\% 2520$ Bogota. doc $+\& \mathrm{~cd}=2 \& \mathrm{hl}=\mathrm{e}$ $\mathrm{s}-419 \& \mathrm{ct}=\mathrm{clnk} \& \mathrm{gl}=\mathrm{cO}$ 